\title{
Optimization of Extraction and Enrichment of Steroidal Alkaloids from Bulbs of Cultivated Fritillaria cirrhosa
}

\author{
Dongdong Wang, Shu Wang, Qingdan Du, Nanyi Wang, Simei Liu, Xiaoxia Wang, \\ and Jinghui Jiang
} Department of Pharmacognosy, West China College of Pharmacy, Sichuan University, No. 17, Duan 3, Renmin Nan Road,
Chengdu 610041, China

Correspondence should be addressed to Dongdong Wang; wddong1988@hotmail.com and Shu Wang; huaxiyaoxue3000@gmail.com

Received 15 December 2013; Accepted 1 March 2014; Published 1 April 2014

Academic Editor: John J. Gildea

Copyright (C) 2014 Dongdong Wang et al. This is an open access article distributed under the Creative Commons Attribution License, which permits unrestricted use, distribution, and reproduction in any medium, provided the original work is properly cited.

\begin{abstract}
The bulbs of cultivated Fritillaria cirrhosa (BCFC) are used in China both for food and folk medicine due to its powerful biological activities. The aim of this study is to optimize the extraction and enrichment conditions of alkaloids from BCFC. Firstly, the orthogonal experimental design was used to optimize and evaluate four variables (ethanol concentration, solid-liquid ratio, extraction time, and temperature). Thereafter, resin adsorption was as a means to enrich alkaloids. Among 16 tested resins, $\mathrm{H}$ 103 resin presented higher adsorption capacity and desorption ratio. The equilibrium experimental data of the adsorption of total alkaloids, imperialine, and peimisine were well-fitted to the pseudo-first-order kinetics model, Langmuir and Freundlich isotherms models. Finally, in order to optimize the parameters for purifying alkaloids, dynamic adsorption and desorption tests were carried out. After one run treatment with $\mathrm{H}-103$ resin, the contents of total alkaloids, imperialine, and peimisine in the product were 21.40-, $18.31-$, and 22.88 -fold increased with recovery yields of $94.43 \%, 90.57 \%$, and $96.16 \%$, respectively.
\end{abstract}

\section{Introduction}

Fritillaria cirrhosa, which belongs to the family Lilliaceae, is primarily distributed in the southwestern China. Bulbs of Fritillaria cirrhosa (BFC) have long been used both for food and folk medicine in many Asian countries [1]. The materials from wild collections can be harvested at least for five years [2]. In the past, the original plants of BFC were mainly obtained from their wild species and thus were unable to meet the demands of the industry [3]. In recent years, there is a significant breakthrough in artificial plantation technologies. The bulbs of cultivated Fritillaria cirrhosa (BCFC) have become the mainstream of BFC in market, which provide the adequate resources for further exploitation and utilization of BFC.

Studies on phytochemistry of BFC indicated that chemical components of BFC included steroidal alkaloids, saponins, terpenoids, glycosides, and many other compounds [4]. As revealed by modern studies, steroidal alkaloids were the major biological active compositions of BFC [5]. Pharmacological studies found that alkaloids from BFC exhibited remarkable antitussive, expectorant, antiasthmatic properties [6, 7], hypotensive effects [8], antibacterial activity, antitumor effects [9-11], anti-inflammatory effects $[12,13]$, and so on. However, the content of total alkaloids of BFC is quite low and varies wildly, approximately ranging from $0.02 \%$ to $0.3 \%$, which is regarded as major quality constraint for its applications. To the best of our knowledge, there are few systematic publication focusing on optimization of the extraction and enrichment for large-scale production of alkaloids from BCFC; thus, there is an urgent need to optimize the extracting and enriching conditions.

Orthogonal array design (OAD) has been widely used to optimize the factors which influenced the extraction yield or extract profiles of bioactive components from natural materials [14]. It has been used to optimize experimental conditions with fewer numbers of experiments $[15,16]$. In 
TABLE 1: Factors and levels of orthogonal experimental design.

\begin{tabular}{lcccc}
\hline Levels & Ethanol concentration $(C, \mathrm{v} / \mathrm{v} \%)$ & Solid-liquid ratio $(R, \mathrm{~mL} / \mathrm{g})$ & Extraction time $(t, \mathrm{~min})$ & Temperature $\left(T,{ }^{\circ} \mathrm{C}\right)$ \\
\hline 1 & 80 & $5: 1$ & 60 & 70 \\
2 & 90 & $10: 1$ & 90 & 80 \\
3 & 100 & $15: 1$ & 120 & 90 \\
\hline
\end{tabular}

this study, OAD was also used to optimize the extraction conditions.

There are several conventional methods, such as polyamide chromatography, gel chromatography, and silica gel column, available for the enrichment of active constituents [14]. However, these methods have several disadvantages, including long time consuming poisonous residual solvents and low recoveries [17]. Recently, growing attention has been taken to enrich and purify targeted components from crude biological samples using macroporous resins for their convenience, low operating costs, low solvent consumption, high chemically stability, and easy regeneration [14, 17]. In this study, the absorption and desorption on macroporous resins were utilized for the enrichment of alkaloids from BCFC.

The present study focused on the optimization of extraction and enrichment of alkaloids from BCFC to develop a cost-effective method for large-scale extraction and enrichment of alkaloids. In extraction process, we tried to optimize four independent variables by using OAD. For enrichment of alkaloids, the optimum macroporous resin was screened and the major process parameters were determined. Meanwhile, the experimental isotherm data were analyzed using pseudofirst- and second-order models, the Langmuir and Freundlich equations. Finally, macroporous resin chromatography was used for the enrichment of alkaloids from BCFC.

\section{Materials and Methods}

2.1. Plant Materials and Reagents. BCFC were purchased from the dealers who cultivate the Fritillaria cirrhosa at Chengdu International Trade City Hehuachi Chinese Medicinal Herbal Market (Chengdu, Sichuan province, China) and identified by Professor Shu Wang (Department of Pharmacognosy, West China College of Pharmacy, Sichuan University, Chengdu, China). The sample (Wang 20120510) has been deposited in the pharmacognosy lab of West China College of Pharmacy, Sichuan University. Anhydrous ethanol, ammonia water, ethylenediamine, and chloroform were analytical grade obtained from Tianjing Kemiou Chemical Reagents Co. (Tianjing, China). Acetonitrile of chromatographic grade was purchased from Sigma Aldrich, Inc. (Steinheim, Germany). Imperialine and peimisine were isolated from BFC in our laboratory $[12,13]$. The ultrapure water was prepared by a Milli-Q water system (Millipore, USA).

2.2. Selection of Extraction Parameters. The preprepared powder of BCFC $(50 \mathrm{~g})$ was soaked in $70 \mathrm{~mL}$ ammonia for different times $(0.5 \mathrm{~h}, 1.0 \mathrm{~h}, 1.5 \mathrm{~h}, 2.0 \mathrm{~h}, 3.0 \mathrm{~h}$, and $4.0 \mathrm{~h})$ and then extracted under reflux with different concentrations of ethanol $(50 \%, 60 \%, 70 \%, 80 \%, 90 \%$, and $100 \%)$ with different ratios of liquid/solid (mL:g) $(5: 1,10: 1,15: 1,20: 1,25: 1$, and $30: 1)$ for different given times $(60 \mathrm{~min}, 90 \mathrm{~min}, 120 \mathrm{~min}$, $150 \mathrm{~min}, 180 \mathrm{~min}$ and $240 \mathrm{~min}$ ), while the temperature of the water bath was set as $50^{\circ} \mathrm{C}, 60^{\circ} \mathrm{C}, 70^{\circ} \mathrm{C}, 80^{\circ} \mathrm{C}, 90^{\circ} \mathrm{C}$, and $95^{\circ} \mathrm{C}$, respectively, and kept steady (within $\pm 2.0^{\circ} \mathrm{C}$ ), for different extraction times (once, twice, thrice, and four times) [18].

\subsection{Orthogonal Experimental Design of Extraction Procedure.} Based on single-factor experimental results, four independent parameters (ethanol concentration $(\mathrm{v} / \mathrm{v}, C)$, solid-liquid ratio $(\mathrm{mL} / \mathrm{g}, R)$, extraction time $(\mathrm{min}, t)$, and temperature $\left({ }^{\circ} \mathrm{C}, \mathrm{T}\right)$ were confirmed as the major influencing factors; then a $\mathrm{L}_{9}\left(3^{4}\right)$ orthogonal experimental design was conducted to evaluate the effects of four independent variables on the extraction efficiency of total alkaloids, which was reflected by extraction yield of total alkaloids of BCFC. The range and levels of individual variables are shown in Table 1. The experiment design is shown in Table 2, along with experimental data. All experiments were run in twice.

2.4. Preparation of Sample Solution and Absorbents. The extract process was done according to optimal extraction conditions obtained in Section 2.3. The extracting solution was filtered, the filtrates were combined and evaporated using a vacuum rotary evaporator at $45^{\circ} \mathrm{C}$ and dried in vacuo for $72 \mathrm{~h}$ to yield ethanol extracts. The sample solution was prepared by dissolving the dried ethanol extracts in distilled water. The physical properties of the 16 kinds of adsorbents are summarized in Table 3. For prior use, the adsorbents needed to be soaked for $24 \mathrm{~h}$ with $100 \%$ ethanol and then washed with $1 \mathrm{~mol} / \mathrm{L} \mathrm{HCl}$ and $\mathrm{NaOH}$ solution successively and finally washed with distilled water to remove monomers and porogenic agents trapped inside the pores during the synthesis process [17].

\subsection{Adsorption Resins Screening}

2.5.1. Adsorption Resins Screening. The optimum resin was screened by static adsorption and desorption tests. The adsorption tests on different resins were performed as follows: preweighed amounts of hydrated resins (equal to $0.5 \mathrm{~g}$ dry resin) and $100 \mathrm{~mL}$ sample solution $(0.3271 \mathrm{mg} / \mathrm{mL})$ were added into $250 \mathrm{~mL}$ flasks which were continually shaken for $24 \mathrm{~h}$ at $30^{\circ} \mathrm{C}$ with $120 \mathrm{rpm}$. The solutions after adsorption were separated from the resins and analyzed by Alpha-1900PC UVVis spectrophotometer and Shimadzu-10AT HPLC coupled to Sedex-75 evaporative light-scattering detector (HPLCELSD) according to the method described previously $[19,20]$. The static desorption tests were conducted as follows: after 
TABLE 2: Orthogonal experimental design for extraction of total alkaloids from BCFC.

\begin{tabular}{|c|c|c|c|c|c|}
\hline Test no. & $t(\min )$ & $C(\mathrm{v} / \mathrm{v} \%)$ & $T\left({ }^{\circ} \mathrm{C}\right)$ & $R(\mathrm{~mL} / \mathrm{g})$ & $Y(\%)$ \\
\hline 1 & 1 & 1 & 1 & 1 & $81.42 \pm 2.78$ \\
\hline 2 & 1 & 2 & 2 & 2 & $96.63 \pm 0.98$ \\
\hline 3 & 1 & 3 & 3 & 3 & $63.81 \pm 4.65$ \\
\hline 4 & 2 & 1 & 2 & 3 & $107.41 \pm 3.28$ \\
\hline 5 & 2 & 2 & 3 & 1 & $80.95 \pm 6.62$ \\
\hline 6 & 2 & 3 & 1 & 2 & $81.73 \pm 3.78$ \\
\hline 7 & 3 & 1 & 3 & 2 & $91.99 \pm 4.80$ \\
\hline 8 & 3 & 2 & 1 & 3 & $106.73 \pm 3.64$ \\
\hline 9 & 3 & 3 & 2 & 1 & $78.84 \pm 2.15$ \\
\hline$k_{1}$ & 80.62 & 93.61 & 89.96 & 80.41 & \\
\hline$k_{2}$ & 90.03 & 94.77 & 94.29 & 90.11 & \\
\hline$k_{3}$ & 92.52 & 74.80 & 78.92 & 92.65 & \\
\hline$R$ & 11.90 & 19.98 & 15.38 & 12.25 & \\
\hline
\end{tabular}

$Y$ : mean value of extraction yield of total alkaloids; $R$ is the difference between the maximum value and the minimum value of $k_{i}$ of any columns. The reported values are expressed as mean \pm S.D. $(n=2)$.

TABle 3: Physical properties of the macroporous resins.

\begin{tabular}{|c|c|c|c|c|}
\hline Name & Polarity & Particle diameter $(\mathrm{mm})$ & Surface area $\left(\mathrm{m}^{2} / \mathrm{g}\right)$ & Average pore diameter $(\mathrm{nm})$ \\
\hline HPD-100 & Nonpolar & $0.3-1.20$ & $650-700$ & $8.5-9.0$ \\
\hline HPD-200A & Nonpolar & $0.3-1.25$ & $700-750$ & $8.5-9.0$ \\
\hline HPD-300 & Nonpolar & $0.3-1.20$ & $800-870$ & $5.0-5.5$ \\
\hline HPD-700 & Nonpolar & $0.3-1.20$ & $650-700$ & $8.5-9.0$ \\
\hline D-101 & Nonpolar & $0.2-0.60$ & $400-600$ & $10.0-12.0$ \\
\hline D-3520 & Nonpolar & $0.3-1.25$ & $480-520$ & $8.5-9.0$ \\
\hline D-4006 & Nonpolar & $0.3-1.25$ & $400-440$ & $6.5-7.5$ \\
\hline H-103 & Nonpolar & $0.3-1.25$ & $900-1100$ & $8.4-9.4$ \\
\hline HPD-450A & Middle-polar & $0.3-1.25$ & $500-550$ & $9.0-10.0$ \\
\hline HPD-750 & Middle-polar & $0.3-1.20$ & $650-700$ & $8.5-9.0$ \\
\hline ADS-17 & Middle-polar & $0.3-1.25$ & $90-150$ & $20.0-30.0$ \\
\hline DM-130 & Middle-polar & $0.3-1.25$ & $500-550$ & $9.0-10.0$ \\
\hline HPD-722 & Weak-polar & $0.3-1.25$ & $485-530$ & $13.0-14.0$ \\
\hline AB-8 & Weak-polar & $0.3-1.25$ & $480-520$ & $13.0-14.0$ \\
\hline HPD-600 & Polar & $0.3-1.20$ & $550-600$ & 8.0 \\
\hline HPD-826 & Polar & $0.3-1.25$ & $500-600$ & $9.0-10.0$ \\
\hline
\end{tabular}

reaching adsorption equilibrium, the resins were washed with $100 \mathrm{~mL}$ distilled water and then desorbed with $50 \mathrm{~mL} 80 \%$ ethanol solution. The flasks were continually shaken for $12 \mathrm{~h}$ at $30^{\circ} \mathrm{C}$ with $120 \mathrm{rpm}$. The adsorption and desorption properties of different resins for alkaloids including adsorption capacity, desorption capacity, and desorption ratio of each resin were quantified with the following equations $[14,21,22]$ :

$$
\begin{gathered}
Q_{e}=\left(C_{0}-C_{e}\right) \times \frac{V_{i}}{W}, \\
Q_{d}=\frac{C_{d} \times V_{d}}{W}, \\
D=\frac{C_{d} \times V_{d}}{\left[\left(C_{0}-C_{e}\right) \times V_{i}\right]} \times 100,
\end{gathered}
$$

where $Q_{e}$ is the adsorption capacity at adsorption equilibrium (mg/g dry resin); $Q_{d}$ is the desorption capacity after desorption equilibrium ( $\mathrm{mg} / \mathrm{g}$ dry resin); $D$ is the desorption ratio (\%); $C_{0}, C_{e}$, and $C_{d}$ are the initial, absorption equilibrium, and desorption concentrations of alkaloids in the solutions, respectively (mg/mL); $V_{i}$ and $V_{d}$ are the volume of the initial sample and desorption solution $(\mathrm{mL})$, respectively; and $W$ is the dry weight of tested resins ( $\mathrm{g}$ ).

To study the adsorption and desorption properties of the selected resin under different conditions, a series of sample solutions with $\mathrm{pH}$ values ranged from 5 to 10 for adsorption were tested, and a series of concentration of ethanol ranged from $10 \%$ to $100 \%$ for desorption were also investigated. All the solutions after adsorption and desorption at different conditions were analyzed by UV-Vis spectrophotometer [23]. 
2.5.2. Adsorption Kinetics. Adsorption kinetics tests were conducted by mixing $0.5 \mathrm{~g}$ (dry weight) of hydrated $\mathrm{H}$ 103 resin with $100 \mathrm{~mL}$ of sample solutions $(0.3078 \mathrm{mg} / \mathrm{mL})$ in flasks. The concentrations of target compounds in the adsorption solution were monitored at different time intervals until equilibrium. Two most widely used adsorption models, pseudo-first-order and pseudo-second-order model, were used in determining the rate of the adsorption process to investigate the adsorption process of alkaloids of BCFC on H-103 resin [14, 21, 24].

The pseudo-first-order equation is given as follows:

$$
\log \left(Q_{e}-Q_{t}\right)=\log \left(Q_{e}\right)-\left(\frac{k_{1} \times t}{2.303}\right)
$$

where $Q_{e}$ and $Q_{t}$ are the amounts of analyte $(\mathrm{mg} / \mathrm{g})$ adsorbed on the resin at equilibrium and at time $t$, respectively, and $k_{1}$ is the rate constant of pseudo-first-order sorption. lows:

The pseudo-second-order equation is expressed as fol-

$$
\frac{t}{Q_{t}}=\frac{1}{k_{2} \times Q_{e}^{2}}+\frac{t}{Q_{e}}
$$

where $k_{2}$ is the rate constant of pseudo-second-order adsorption; $Q_{e}$ and $Q_{t}$ are the same as described above.

The linear intraparticle diffusion equation was further fitted using the adsorption kinetic data to determine whether intraparticle diffusion is the rate-limiting step [24]. The intraparticle diffusion equation is given as follows:

$$
Q_{t}=k_{i} \times t^{1 / 2}+C
$$

where $k_{i}$ and $C$ are the intraparticle diffusion rate constants; $Q_{t}$ is the same as described above.

2.5.3. Adsorption Isotherms. Afterwards, the tests for equilibrium adsorption isotherms on the selected resin were performed. $100 \mathrm{~mL}$ of sample solutions at different concentrations and preweighed amounts of hydrated resins (equal to $0.1 \mathrm{~g}$ dry resin) were added into $250 \mathrm{~mL}$ flasks and shaken for $24 \mathrm{~h}$ at different temperatures $\left(20,30\right.$, and $\left.40^{\circ} \mathrm{C}\right)$. The effects of the initial concentration of sample solution and temperature on the adsorption characteristics were studied with the thermodynamic parameters of adsorption determined. Two standard theoretical models, well-known Langmuir model and Freundlich model, were frequently used to describe the adsorption behavior [21, 24].

The Langmuir isotherm equation is expressed as follows:

$$
\frac{C_{e}}{Q_{e}}=\frac{C_{e}}{Q_{0}}+\frac{1}{K \times Q_{0}},
$$

where $Q_{0}$ is an empirical constant and $K$ is the Langmuir adsorption equilibrium constant. $C_{e}$ and $Q_{e}$ are the same as described above.

The Freundlich isotherm equation is given as follows:

$$
\ln Q_{e}=\ln k_{f}+\frac{1}{n} \times \ln C_{e}
$$

where $1 / n$ is an empirical constant and $k_{f}$ is the Freundlich adsorption equilibrium constant [24]. $C_{e}$ and $Q_{e}$ are the same as described above.

The Gibbs free energy $\left(\Delta G^{0}\right)$ change indicates the degree of the spontaneity of the adsorption process [24]. The equation for $\Delta G^{0}$ is defined as follows:

$$
\begin{gathered}
\Delta G^{0}=-R \times T \times \ln k, \\
\ln k=\frac{-\Delta H^{0}}{R \times T}+\frac{\Delta S^{0}}{R},
\end{gathered}
$$

where $T$ is temperature $(K), \Delta H^{0}$ is the enthalpy of adsorption $(\mathrm{kJ} / \mathrm{mol}), \Delta S^{0}$ is the entropy of adsorption $(\mathrm{J} / \mathrm{mol} \cdot \mathrm{K})$, and $k$ is the equilibrium constant obtained from the best fitted model at 293,303 , or $313 \mathrm{~K}$.

\subsection{Dynamic Adsorption and Desorption Tests}

2.6.1. Effect of Concentration of Alkaloids in Sample Solution on Dynamic Adsorption. Dynamic adsorption tests were first carried out on glass columns $(250 \mathrm{~mm} \times 15 \mathrm{~mm}$ i.d. $)$ wetpacked H-103 resin with the bed volume (BV) of $8 \mathrm{~mL}$. The sample solution was loaded onto the macroporous resin columns at different concentrations of the loading sample solution $(0.10,0.20$, and $0.40 \mathrm{mg} / \mathrm{mL})$. The sample solution flowed through the resin column at a constant flow rate of 1.0 BV/h. The concentration of alkaloids in the aliquots of $1 \mathrm{~mL}$ effluents collected at 3.0 BV interval was monitored by $\mathrm{UV}-\mathrm{Vis}$ spectrophotometer to get the dynamic breakthrough curve and select the most suitable concentration of the loading sample solution.

2.6.2. Effect of Diameter to Height Ratio on Dynamic Adsorption. Besides, the effect of diameter-to-height ratio on breakthrough curve was examined in a similar way. The sample solution flowed through the columns (diameter-to-height ratio $1 / 3,1 / 10$, or $1 / 30$ ) at a constant flow rate of $1.0 \mathrm{BV} / \mathrm{h}$ and the concentration of alkaloids in sample solution was approximate $0.20 \mathrm{mg} / \mathrm{mL}$.

2.6.3. Effect of Loading Flow Rate on Dynamic Adsorption. In adsorption process, in addition to concentration of the loading sample solution and diameter-to-height ratio, the effect of loading flow rate on breakthrough curve was also investigated. The sample solution was loaded onto the macroporous resin column (diameter-to-height ratio 1/10) at different flow rates of $2.0,4.0$, and $6.0 \mathrm{BV} / \mathrm{h}$ and the concentration of total alkaloids was approximately $0.20 \mathrm{mg} / \mathrm{mL}$.

2.6.4. Effect of Ethanol-Water Concentrations on Dynamic Desorption. After adsorption equilibrium under the optimum condition, the adsorbate-laden column was first washed with $8 \mathrm{BV}$ of distilled water and $4 \mathrm{BV}$ of $10 \%$ aqueous ethanol solution at a constant flow rate of $2 \mathrm{BV} / \mathrm{h}$ to elute impurities and then eluted with different concentrations of ethanol $(70 \%, 80 \%, 90 \%, 95 \%)$ at the flow rate of $2 \mathrm{BV} / \mathrm{h}$, respectively. 
TABLE 4: Variance analysis of orthogonal experimental data.

\begin{tabular}{|c|c|c|c|c|}
\hline Source of variance & Sum of square & Degree of freedom & Mean square & $F$ value \\
\hline Extraction time & 472.780 & 2 & 236.390 & $15.176^{* *}$ \\
\hline Ethanol concentration & 1508.467 & 2 & 754.233 & $48.422^{* * *}$ \\
\hline Temperature & 754.461 & 2 & 377.231 & $24.218^{* * *}$ \\
\hline Solid-liquid ratio & 501.241 & 2 & 250.620 & $16.090^{* *}$ \\
\hline Error & 140.187 & 9 & 15.576 & \\
\hline Total & 141896.623 & 18 & & \\
\hline
\end{tabular}

$F$ critical value $(95 \%)=4.25$; $^{* *} P<0.01 ;{ }^{* * *} P<0.001$.

2.7. Laboratory Preparative-Scale Purification. The crude extracts from BFC (400.0 g) were dissolved in water. The sample solution ( $\mathrm{pH} 7.0$ ) was applied to a glass column $(120.0 \mathrm{~cm} \times 7.5 \mathrm{~cm}$ i.d.) containing $2.0 \mathrm{~kg}$ of wet $\mathrm{H}-103$ macroporous resin with a bed volume (BV) of $2.5 \mathrm{~L}$. Initially, distilled water was used to wash the elution solution until almost no color was noted, then $4 \mathrm{BV}$ of $10 \%$ ethanol was used to remove the high polar components, and the adsorbent was finally rinsed with $6 \mathrm{BV}$ of $90 \%$ ethanol to obtain the alkaloids-rich fraction. The flow rate of each gradient was set at about $2 \mathrm{BV} / \mathrm{h}$, and the elution of $90 \%$ ethanol was collected, concentrated, and dried.

2.8. Statistical Analysis. The data were presented as means \pm standard deviation (S. D.) and evaluated by one-way analysis of variance (ANOVA) using the Statistical Package for Social Sciences (SPSS) computer software program. $P$ values $<0.05$ were regarded as significant.

\section{Results and Discussion}

3.1. Extraction Parameters. The extraction rate of constituents from crude resources was affected by many factors, such factors as extraction solvent, extraction time, temperature, and the ratio of liquid-solid, which were usually considered to have significant effect on compounds extraction rate $[18,25]$.

All parameters were tested in a wider range prior to OAD optimization which help narrow down the ranges of the parameters tested. The effect of different parameters (the time of BCFC soaked in ammonia, concentrations of alcohol, extraction time, liquid-solid ratio, temperature, and times of extraction) on the extraction yield of alkaloids was shown in Figure 1. The results indicated that each selected parameter, except the time of BCFC soaked in ammonia and times of extraction, had a suitable level, at which the total alkaloids extraction rate reached the peak. It can be easily concluded from the corresponding figures that the extraction time $150 \mathrm{~min}$, liquid-solid ratio $10: 1$, and concentrations of alcohol $80 \%$ were of suitable levels. So, concentrations of alcohol, liquid-solid ratio, extraction time, and temperature were selected as the four extraction parameters for the following OAD [18].

3.2. Optimization of Extraction Conditions. To verify whether the effect of individual factors on total alkaloids extraction efficiency is statistically significant, an ANOVA test was used to analyze the experimental data $[25,26]$. The significance of each factor was evaluated by calculating the $R$ value of extreme difference (Table 2 ) and the $F$ value (Table 4 ). As seen in Tables 2 and 4, we concluded that the order of parameters influencing on extraction rate of total alkaloids was as follows: ethanol concentration $>$ temperature $>$ liquid-solid ratio $>$ extraction time, and the four parameters were all statistically significant. Based on the analysis, the optimum conditions of extraction were therefore determined as follows: ethanol concentration (\%,v/v) $90 \%$, temperature $80^{\circ} \mathrm{C}$, liquid-solid ratio $15: 1$, and extraction time $120 \mathrm{~min}$. In order to validate the optimum conditions, a verification experiment was carried out. Under these conditions an extraction yield of total alkaloids from BCFC of $97.84 \%$ was obtained.

3.3. Screening of Optimum Resin. To obtain the most appropriate resins, absorption and desorption properties of sixteen macroporous resins towards total alkaloids, imperialine, and peimisine were assessed and the results were shown in Table 5. HPD-100, H-103, and HPD-722 resins exhibited notably higher adsorption capacity towards total alkaloids, imperialine, and peimisine than that of other resins. Moreover, the adsorption capacity of alkaloids on $\mathrm{H}-103$ resin was the highest among the three resins. Besides, the desorption capacity and desorption ratio of HPD-722 resin for alkaloids were lower than that of HPD-100 and H-103 and desorption ratio of $\mathrm{H}-103$ resin was close to that of $\mathrm{HPD}-100$. In view of these results, $\mathrm{H}-103$ resin was considered as the optimum one. $\mathrm{H}-103$ resin exhibited better adsorption capacity and desorption ratio not only because of similar polarity with the nonpolar alkaloids from BCFC, but also because of its higher surface area which may correlate with the capability of the resin and the chemical features of the adsorbed substance [21].

\subsection{Effects of $p H$ on the Absorption Capacity and Ethanol} Concentration on the Desorption Capacity. The $\mathrm{pH}$ plays an important role in absorption processes because the ionization of solutes can be influenced by $\mathrm{pH}$, thus affecting the absorption affinity between solutes and the adsorbents [14, 27]. The effect of $\mathrm{pH}$ of initial sample solution on the absorption capacity was shown in Figure 2(a). It shows that for total alkaloids the highest adsorption capacity appeared at the $\mathrm{pH}$ value of 7.0. The reason for the sharp decrease when the $\mathrm{pH}$ value was below 7.0 is that the alkaloids were ionized with decreasing of $\mathrm{pH}$. In addition, increase of $\mathrm{pH}$ produces 


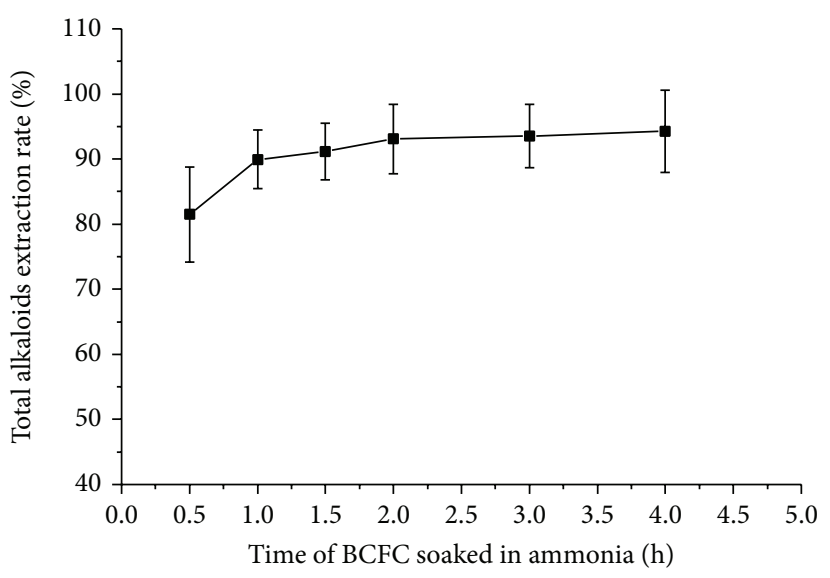

(a)

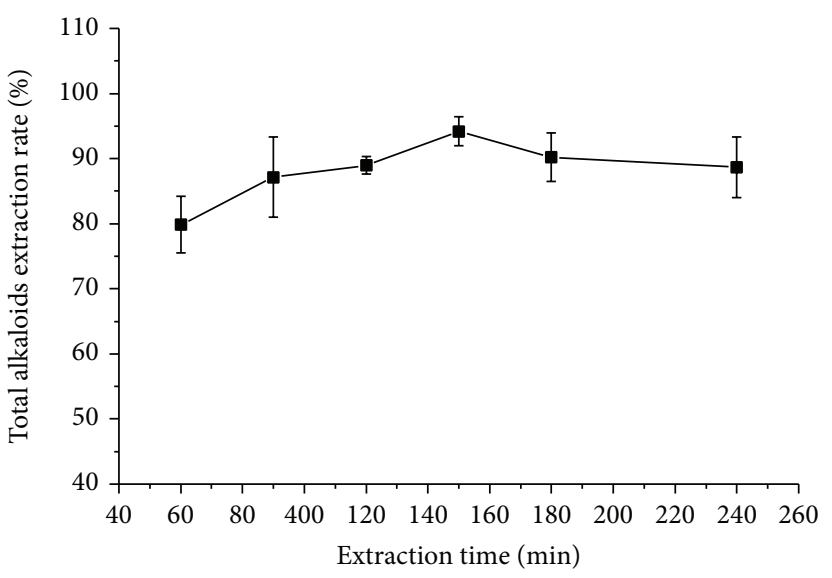

(c)

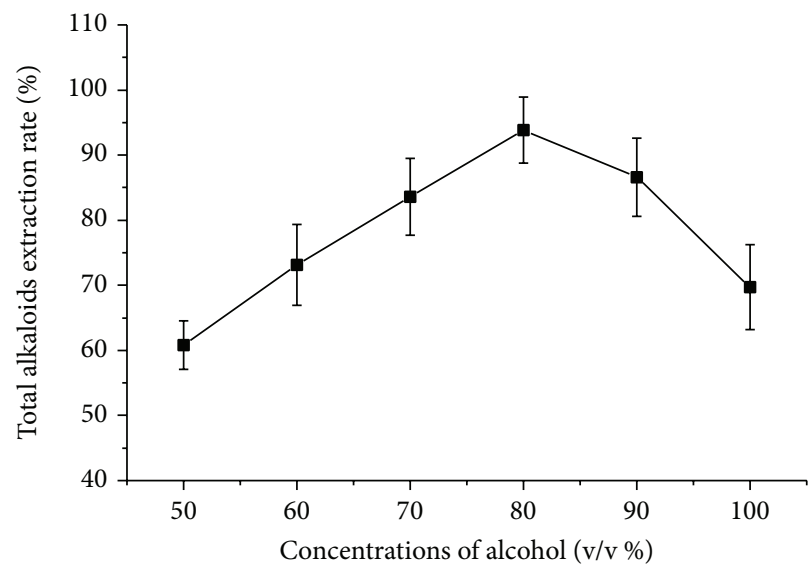

(e)

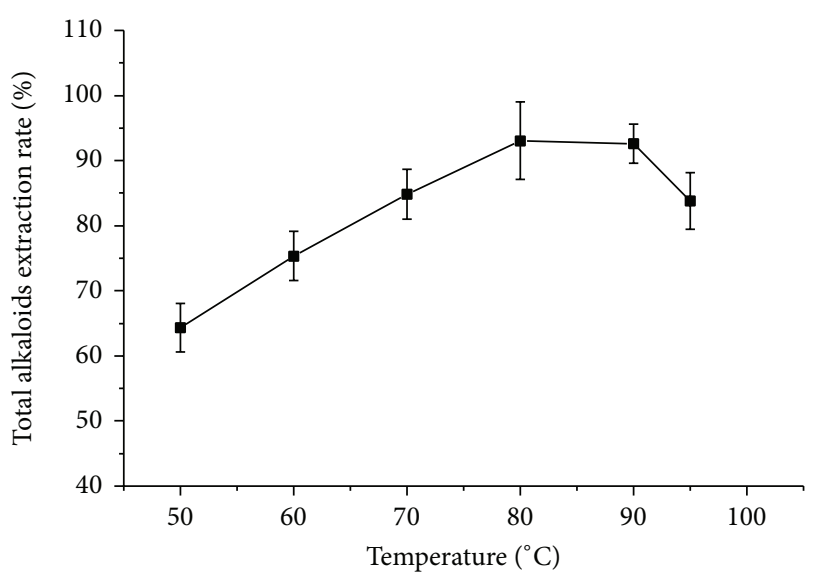

(b)

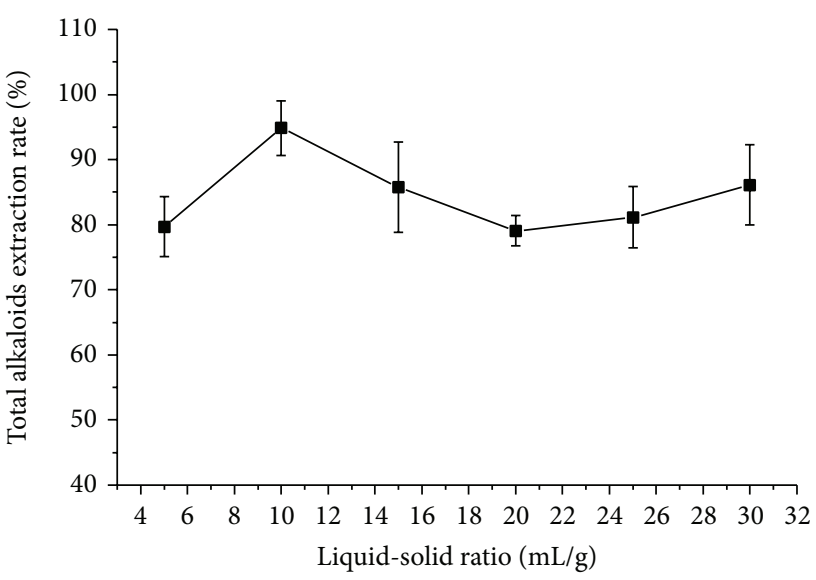

(d)

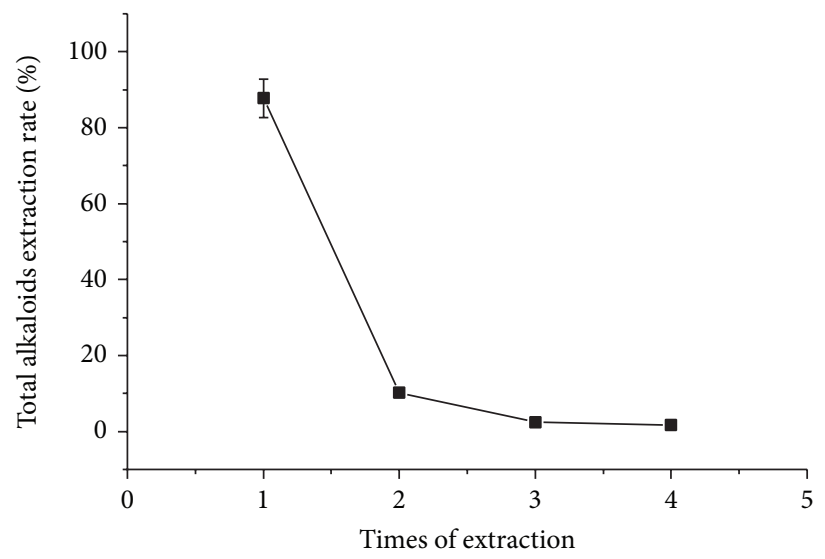

(f)

FIGURE 1: Effects of different parameters: the time of BCFC soaked in ammonia (a), temperature (b), extraction time (c), liquid-solid ratio (d), concentrations of alcohol (e), and times of extraction (f) on the total alkaloids extraction ratio.

a lot of precipitation, which exerted negative influence on the absorption capacity. Based on these results, $\mathrm{pH} 7.0$ of sample solutions was selected for absorption in the following tests.

Different concentrations of ethanol solutions were used to perform desorption experiments in order to choose proper desorption solution. As shown in Figure 2(b), the desorption ratios of alkaloids increased with the increase of ethanol concentration and reached their peak value at the concentration of $80 \%$, then decreased with the increase of ethanol concentration [23]. 


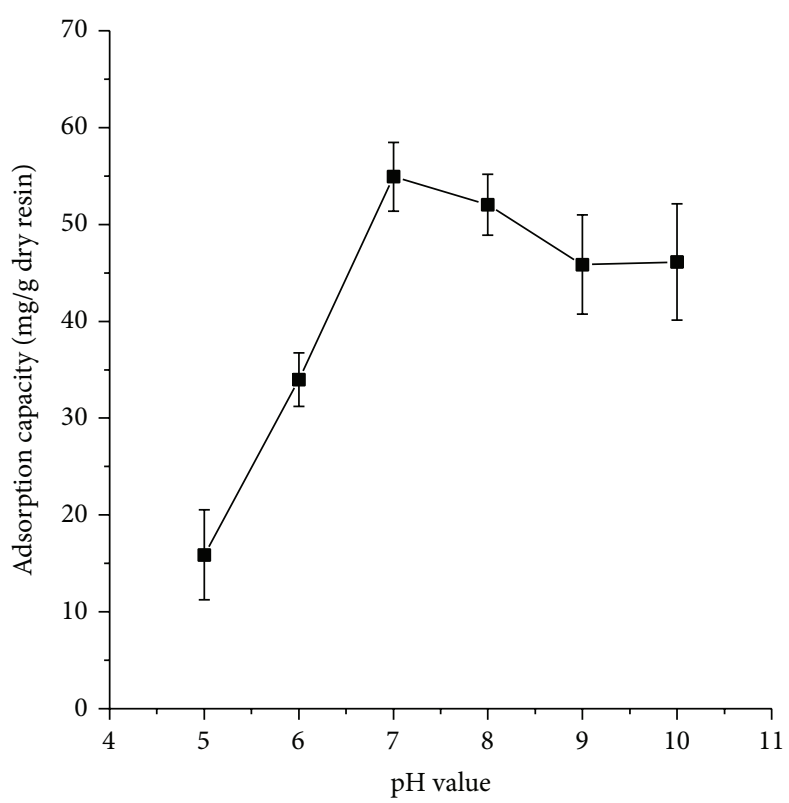

(a)

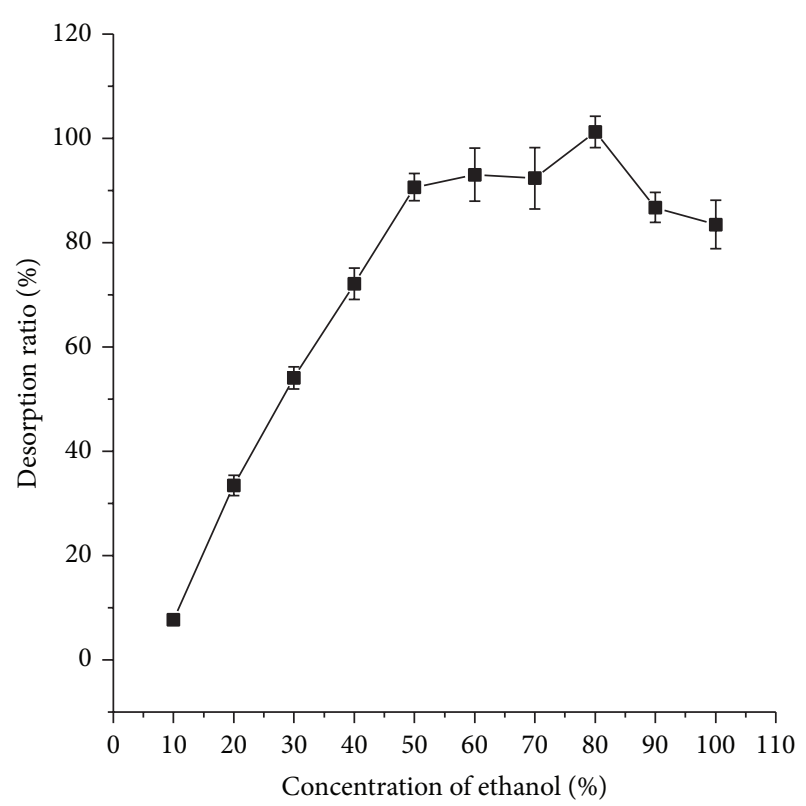

(b)

FIGURE 2: Effects of $\mathrm{pH}$ on the absorption capacity (a) and ethanol concentration on the desorption capacity (b) of total alkaloids.

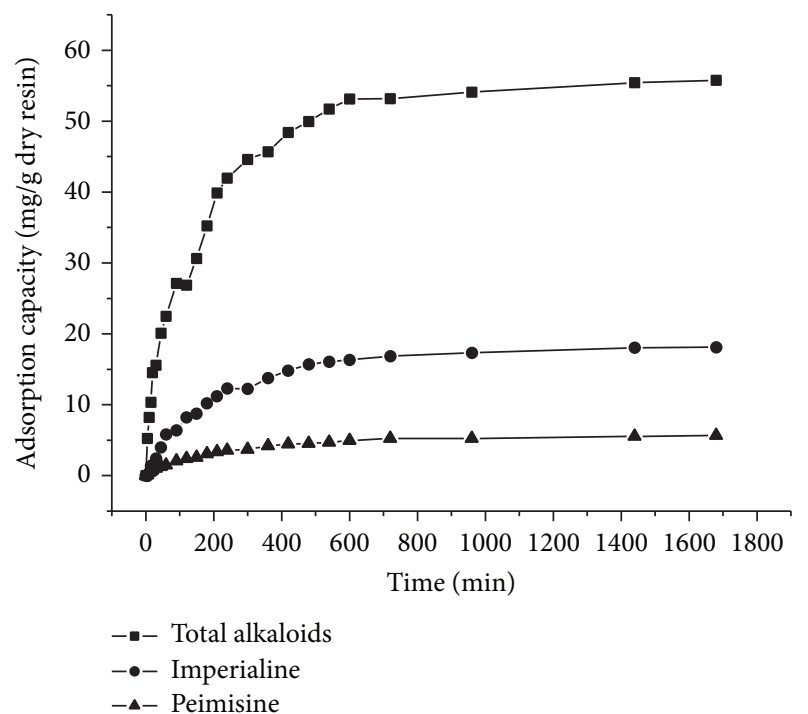

FIGURE 3: Adsorption kinetics curves of total alkaloids, imperialine, and peimisine on $\mathrm{H}-103$ resin.

3.5. Adsorption Kinetics. Adsorption kinetics of total alkaloids, imperialine, and peimisine on $\mathrm{H}-103$ resin were investigated at $30^{\circ} \mathrm{C}$ and the adsorption kinetics curves were obtained. As shown in Figure 3, the adsorption of total alkaloids, imperialine, and peimisine increased with adsorption time before reaching equilibrium. In the first $240 \mathrm{~min}$, the adsorption of total alkaloids rapidly increased and then increased slowly until adsorption equilibrium was reached at $600 \mathrm{~min}$. The adsorption kinetics for imperialine and peimisine showed similar tendency with total alkaloids.
The kinetic parameters and correlation coefficients of the kinetic models were listed in Table 6. As shown in Table 6, the calculated $Q_{e}$ of the pseudo-first-order equation values was closer to the experimental ones, and the correlation coefficients were almost higher than 0.95 . Therefore, the first-order equation described the experimental data more accurately for total alkaloids, imperialine, and peimisine. On the other hand, the correlation coefficients for the pseudosecond-order kinetic model obtained were quite high, but the calculated $Q_{e}$ values deviated significantly from the experimental ones. Thus, the adsorption observed did not fit the pseudo-second-order model.

The intraparticle diffusion model was used to evaluate the possibility of intraparticle diffusion. As shown in Table 6, based on the correlation coefficient of intraparticle diffusion model, $R^{2}$ indicated that pore diffusion was an important rate-limiting step.

3.6. Adsorption Isotherms. The adsorption isotherms were shown in Figure 4. From the adsorption isotherm, the adsorption capacity increased with the initial concentration and reached the saturation plateau when the initial concentrations of total alkaloids, imperialine, and peimisine were 0.204 (Figure 4(a)), 0.074 (Figure 4(b)), and $0.024 \mathrm{mg} / \mathrm{mL}$ (Figure 4(c)), respectively. The adsorption isotherms for imperialine and peimisine showed similar tendency with that of total alkaloids.

The Langmuir and Freundlich equations are used to describe how solutes interact with the resins. The Langmuir model describes the monomolecular layer adsorption without mutual interaction between adsorbed molecules, while the Freundlich model is used to describe the adsorption behavior of monomolecular layer as well as that of 


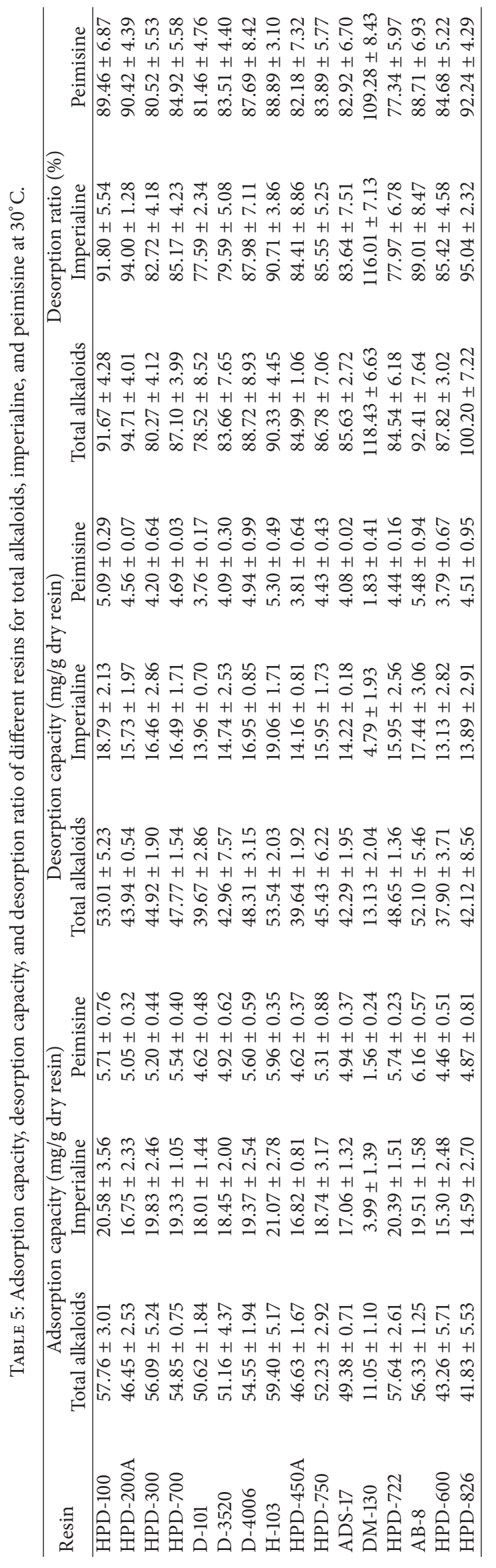




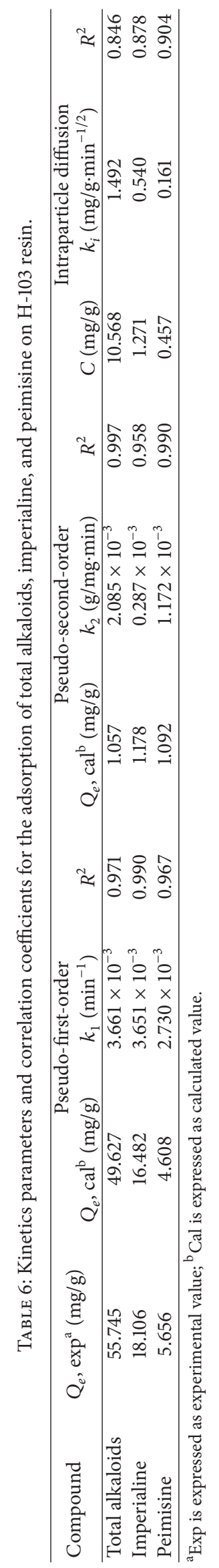




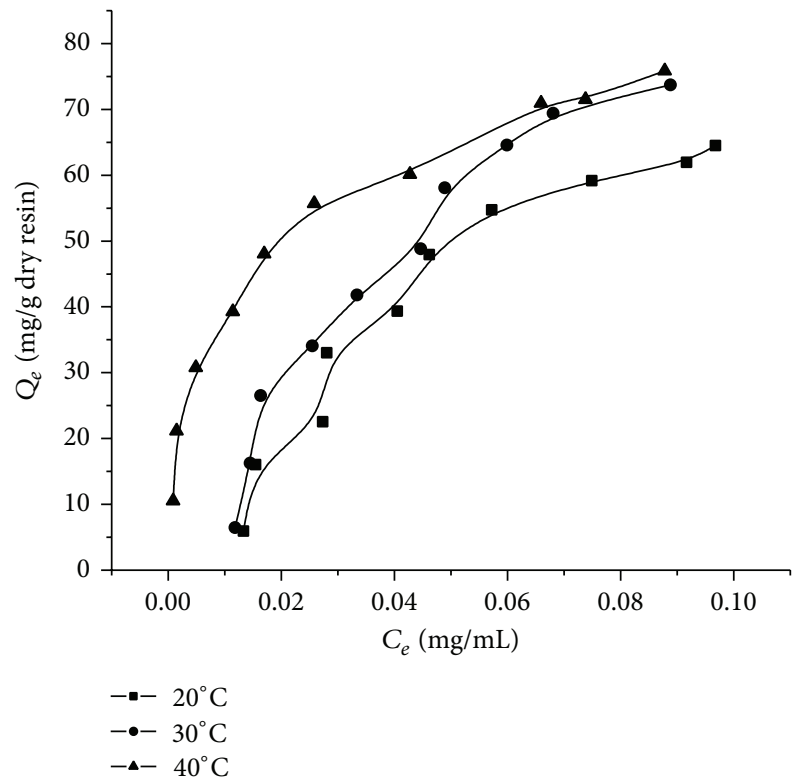

(a)

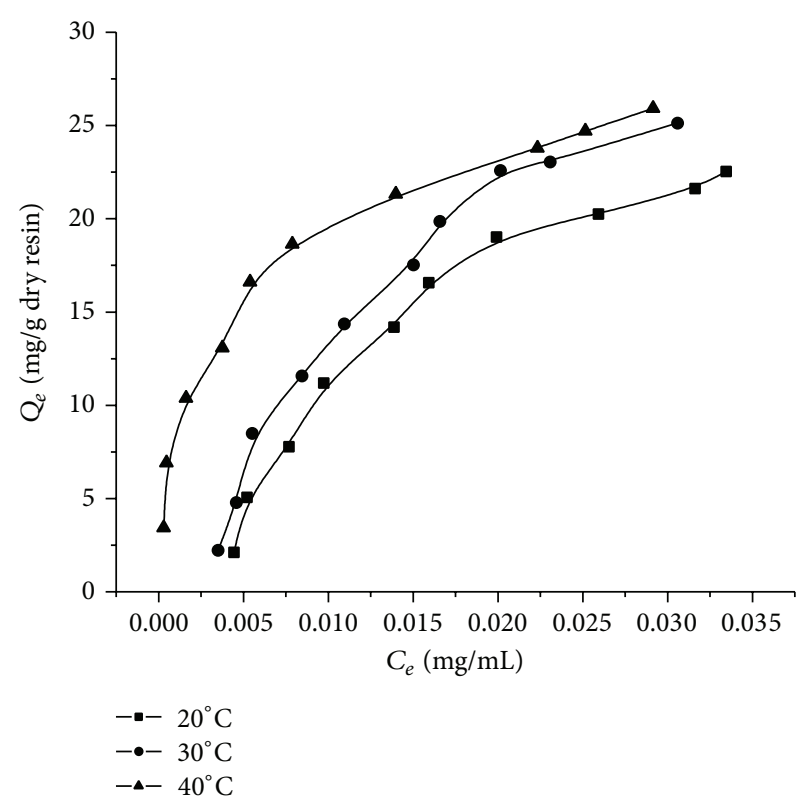

(b)

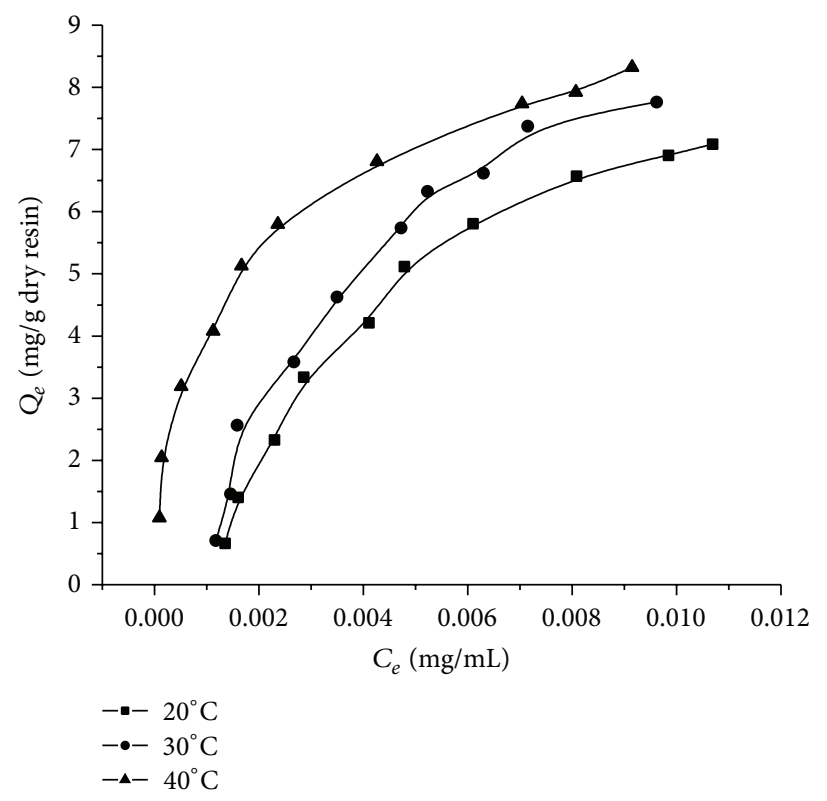

(c)

FIgURE 4: Adsorption isotherms of total alkaloids (a), imperialine (b), and peimisine (c) on $\mathrm{H}-103$ resin at 20,30 , and $40^{\circ} \mathrm{C}$.

the multimolecular layer [28]. As shown in Table 7, the correlation coefficients of both Langmuir and Freundlich equations for total alkaloids, imperialine, and peimisine were rather high. The experimental adsorption data were better fitted to Freundlich equation than Langmuir equation. In the Freundlich equation, values $1 / n<1.0$ represent favorable adsorption conditions and values $1 / n>1.0$ indicates difficulty in adsorption [24]. In Table 7 , the $1 / n$ values are between 0.1 and 1.0, which indicated that the adsorption of total alkaloids, imperialine, and peimisine on $\mathrm{H}-103$ resin can take place easily. We also could see from Figure 4 that at a constant initial concentration, the adsorption capacities increased with temperature increase from 20 to $40^{\circ} \mathrm{C}$, which indicated that the adsorption was an endothermic process.

The thermodynamic parameters of the adsorption process were listed in Table $8 . \Delta G^{0}$ values were negative, indicating that the feasibility and spontaneity of the adsorption process. Its absolute values were less than the critical value $(20 \mathrm{~kJ} / \mathrm{mol})$, showing that the adsorption process was physical $[24,29]$. 


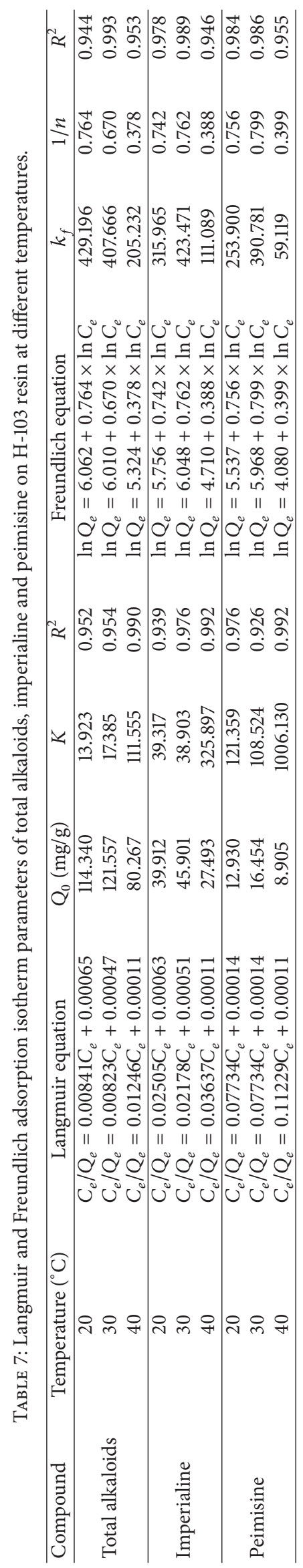


TABLE 8: The thermodynamic parameters of adsorption of total alkaloids, imperialine, and peimisine on $\mathrm{H}-103$ resin at different temperatures.

\begin{tabular}{|c|c|c|c|c|c|c|}
\hline \multirow{2}{*}{ Compound } & \multicolumn{3}{|c|}{$k(\mathrm{~L} / \mathrm{mg})$} & \multicolumn{3}{|c|}{$\Delta G^{0}(\mathrm{~kJ} / \mathrm{mol})$} \\
\hline & $293 \mathrm{~K}$ & $303 \mathrm{~K}$ & $313 \mathrm{~K}$ & $293 \mathrm{~K}$ & $303 \mathrm{~K}$ & $313 \mathrm{~K}$ \\
\hline Total alkaloids & 13.923 & 39.317 & 121.359 & -6.415 & -7.194 & -12.268 \\
\hline Imperialine & 17.385 & 38.903 & 108.524 & -8.944 & -9.223 & -15.058 \\
\hline Peimisine & 111.555 & 325.897 & 1006.130 & -11.690 & -11.807 & -17.992 \\
\hline
\end{tabular}
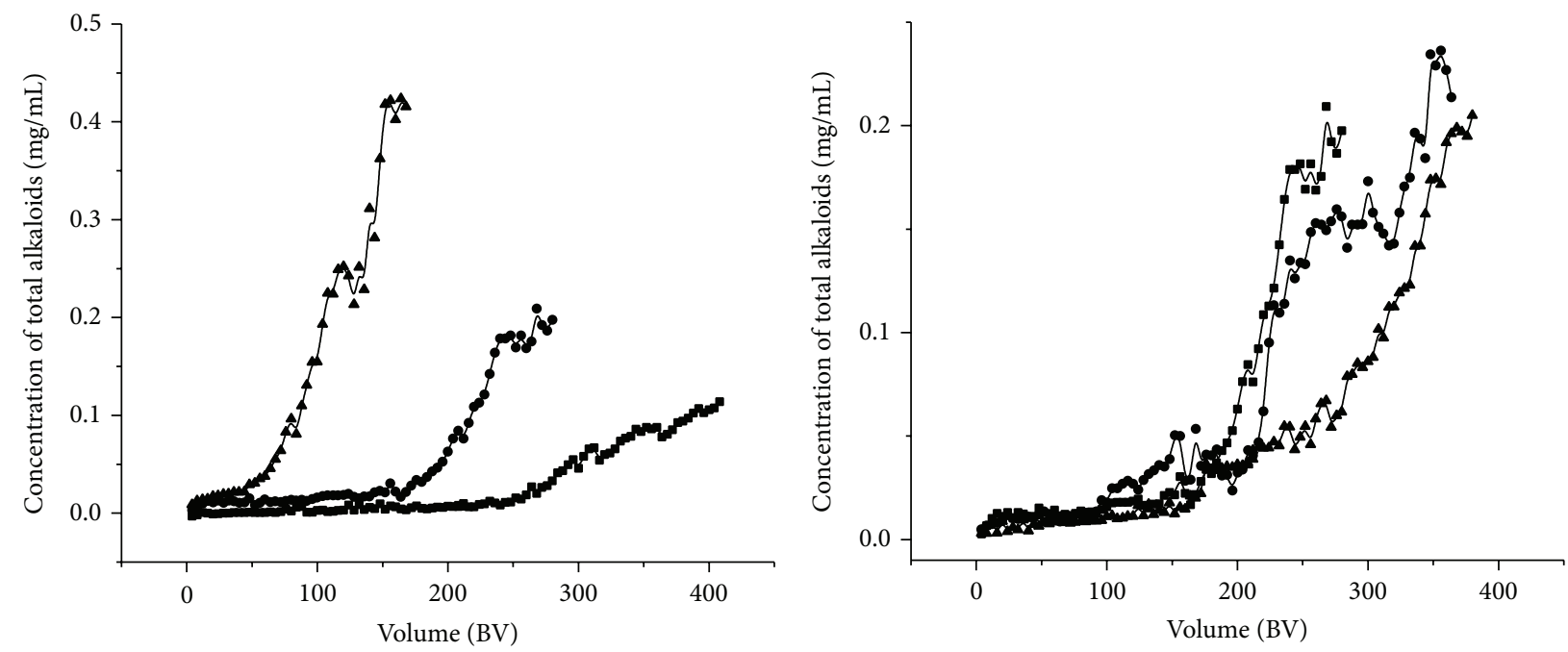

$\begin{array}{ll}- & 0.1 \mathrm{mg} / \mathrm{mL} \\ \text {-•- } 0.2 \mathrm{mg} / \mathrm{mL} \\ \text {-ஃ- } 0.4 \mathrm{mg} / \mathrm{mL}\end{array}$

$-1 / 3$

$-\cdot-1 / 10$

$-1 / 30$

(a)

(b)

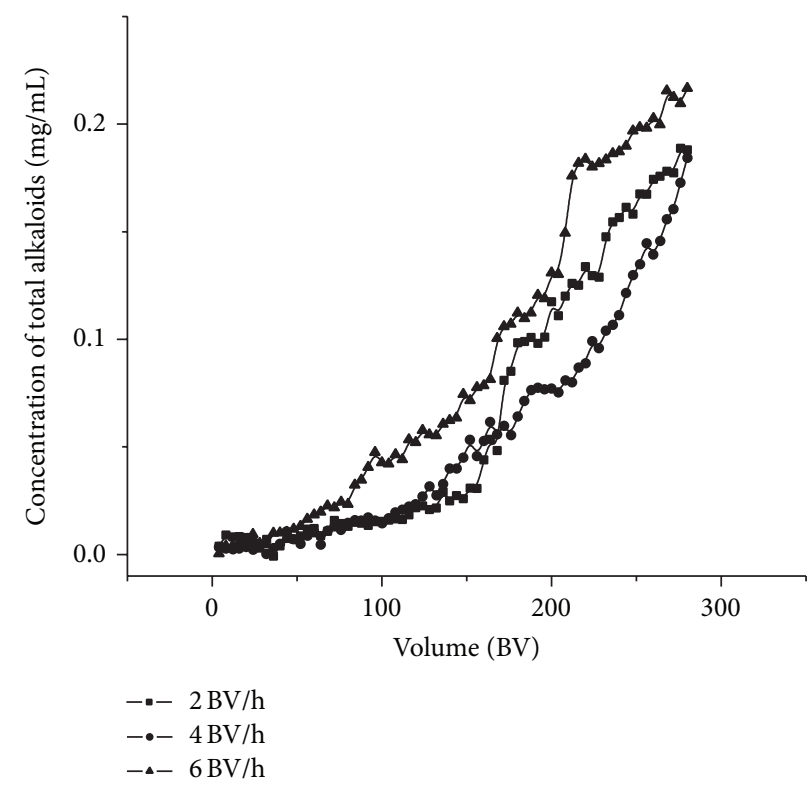

(c)

FIGURE 5: Dynamic breakthrough curves of total alkaloids on column packed with H-103 resin at different concentrations of sample (a), different diameter-to-height ratios (b), and at different sample flow rates (c). 


\subsection{Dynamic Adsorption and Desorption on H-103 Resin}

3.7.1. Dynamic Leakage Curves on H-103 Resin. Adsorption presumably reached equilibrium when the leakage solution concentration was $5 \%$ or $10 \%$ of the initial concentration, which defined the leakage point $[24,30]$. The breakthrough point in this study was set as $5 \%$ of the initial concentration.

As shown in Figure 5(a), the adsorption capacities at the leakage point were $118.24,108.99$, and $92.16 \mathrm{mg} / 8 \mathrm{~mL}$ resin at the concentrations of $0.10,0.20$, and $0.40 \mathrm{mg} / \mathrm{mL}$, respectively. The adsorption capacities at the leakage point increased a little as the concentration decreased from 0.20 to $0.10 \mathrm{mg} / \mathrm{mL}$, but increased significantly as the concentration decreased from 0.40 to $0.20 \mathrm{mg} / \mathrm{mL}$. So the appropriate concentration of total alkaloids during the loading of the sample on the column was set as $0.20 \mathrm{mg} / \mathrm{mL}$.

As shown in Figure 5(b), the adsorption capacities at the leakage point were $108.99,143.76$, and $162.95 \mathrm{mg} / 8 \mathrm{~mL}$ resin at the diameter to height ratio of $1 / 3,1 / 10$, and $1 / 30$, respectively. The adsorption capacities increased significantly when the diameter-to-height ratio increased from $1 / 3$ to $1 / 30$. The significant increase in adsorption capacities was not observed when the diameter-to-height ratio increased from $1 / 10$ to $1 / 30$. Moreover, excessively more ratio of diameterto-height was not conducive to large-scale application. So, a moderate diameter to height ratio of $1 / 10$ was used in the following tests.

As shown in Figure 5(c), the adsorption capacities at the leakage point were $140.88,110.81$, and $83.42 \mathrm{mg} / 8 \mathrm{~mL}$ resin at the flow rate of 2,4 , and $6 \mathrm{BV} / \mathrm{h}$, respectively. With the increase of the sample flow rate, the adsorption capacities at the leakage point reduced because the interaction time of alkaloids with active sites of the resin surface is decreased with the increase of sample flow rate [31]. The adsorption ratio was highest at a flow rate of $2.0 \mathrm{BV} / \mathrm{h}$. Considering that the lower flow rate gave a long run time and was not conducive to industrial production, the flow rate for loading sample was maintained constantly at $4.0 \mathrm{BV} / \mathrm{h}$. According to the above experiments, the corresponding breakthrough volumes of alkaloids on $\mathrm{H}-103$ resin column of diameter-to-height ratio $1 / 10$ were $88 \mathrm{BV}$ at the concentrations of $0.2 \mathrm{mg} / \mathrm{mL}$, at the flow rate of $4.0 \mathrm{BV} / \mathrm{h}$ (Figure 5(c)).

3.7.2. Dynamic Desorption Curves on H-103 Resin. As shown in Section 3.4, at the $10 \%$ ethanol, alkaloids were hardly desorbed. The desorption ratios of total alkaloids reached their peak value at the concentration of $80 \%$ in static desorption. Therefore, we used different concentrations of ethanol $(70 \%, 80 \%, 90 \%$, and $95 \%)$ to establish the proper desorption condition on dynamic desorption. The results were shown in Figure 6. Samples after purification by H-103 resin were combined, concentrated in a rotary evaporator, and dried under vacuum. After dynamic desorption on $\mathrm{H}-103$ resin column chromatography, the contents of total alkaloids in the products which were eluted by $70 \%, 80 \%, 90 \%$, and $95 \%$ ethanol were $486.14,550.89,638.48$, and $643.20 \mathrm{mg} / \mathrm{g}$, respectively, which were 17.23-, 19.53-, 22.63-, and 22.80-fold increased. Their recoveries which were eluted by $70 \%, 80 \%$, $90 \%$, and $95 \%$ ethanol were $95.75 \%, 96.64 \%, 95.87 \%$, and

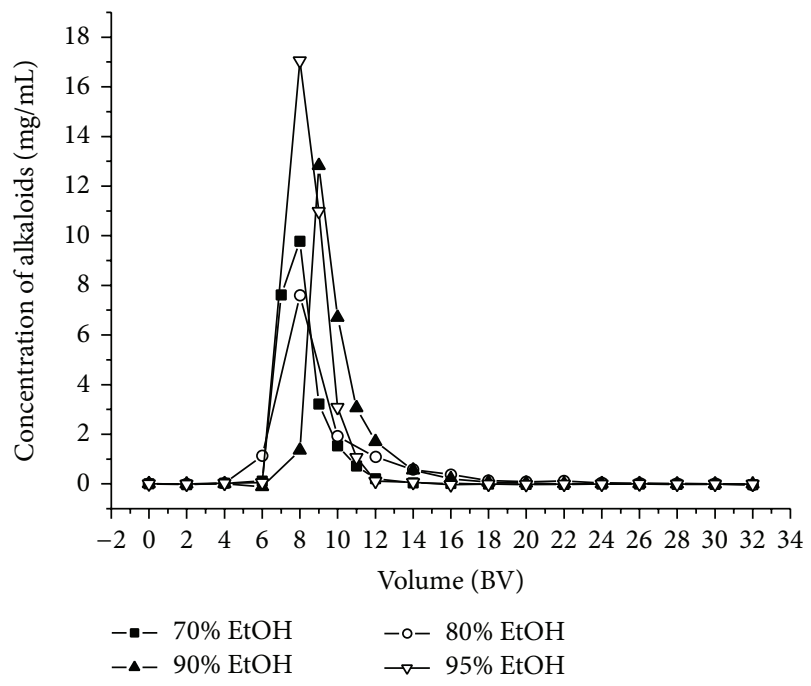

FIGURE 6: Dynamic desorption curves of total alkaloids on column packed with $\mathrm{H}-103$ resin by different concentrations of ethanol solutions.

95.46\%, respectively. Considering efficiency and economy of the process, $90 \%$ ethanol was selected as the desorption solution in the dynamic desorption experiment. Thus, the optimal enrichment conditions for alkaloids on $\mathrm{H}-103$ resin were as follows: for adsorption: initial concentrations of total alkaloids in sample solution: $0.20 \mathrm{mg} / \mathrm{mL}$, diameter-toheight ratio of resin column: $1 / 10$, loading flow rate: $2 \mathrm{BV} / \mathrm{h}$, feed volume: $88 \mathrm{BV}$, and $\mathrm{pH} 7.0$; temperature: $40^{\circ} \mathrm{C}$ and for desorption: a successive gradient elution: $8 \mathrm{BV}$ distilled water, $4 \mathrm{BV} \mathrm{10 \%} \mathrm{ethanol,} 6 \mathrm{BV} 90 \%$ ethanol, and flow rate: $2 \mathrm{BV} / \mathrm{h}$.

3.8. Laboratory Preparative-Scale Purification. All methods used for purifying alkaloids from BCFC were investigated in a small-scale preparation. This was followed by a largescale preparation of pure alkaloids using the above determined conditions. The elution of $6 \mathrm{BV} 90 \%$ ethanol gave the alkaloids-rich fraction, in which the contents of total alkaloids, imperialine, and peimisine were $21.40-, 18.31-$, and 22.88 -fold increased with recovery yields of $94.43 \%, 90.57 \%$, and $96.16 \%$, respectively. The HPLC chromatograms of the experimental samples before and after purification with $\mathrm{H}$ 103 resin were shown in Figure 7.

\section{Conclusions}

The present study established an effective extraction and enrichment procedure for alkaloids from BCFC. On the one hand, the extraction of alkaloids from BCFC was investigated with a four-variable, three-level experiment orthogonal experimental design in enhancing the alkaloids extraction yield. On the other hand, this study reports resin adsorption as a means to enrich alkaloids from BCFC extracts was successfully developed. Among 16 tested resins, H-103 resin presented higher adsorption capacity and desorption ratio. The equilibrium experimental data of the adsorption of total 


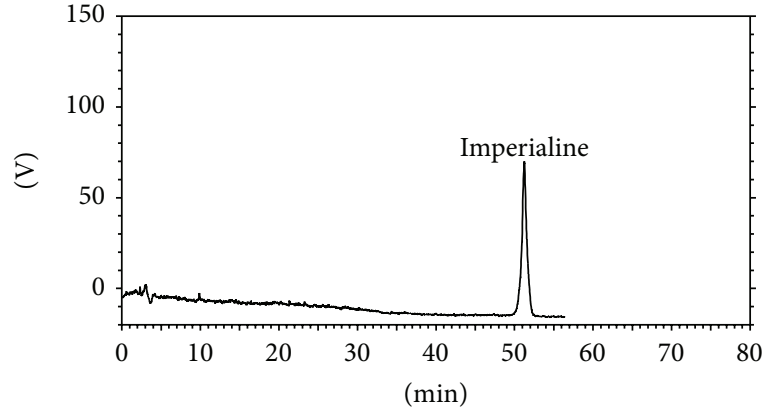

(a)

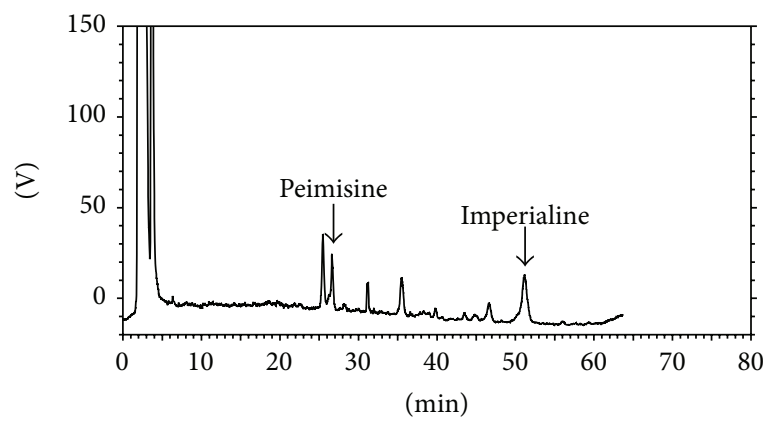

(c)

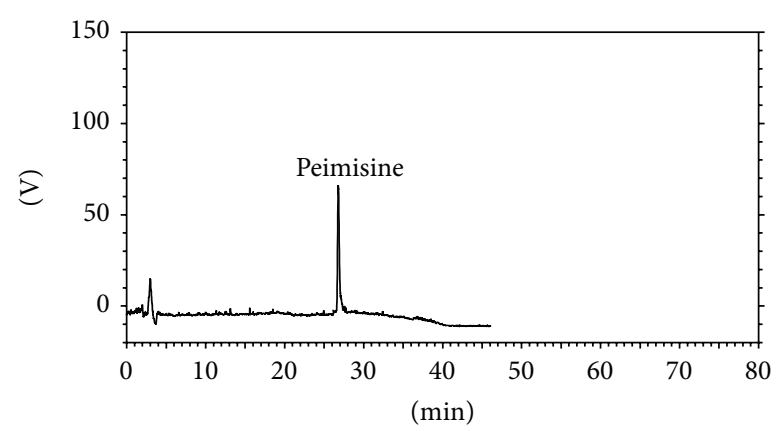

(b)

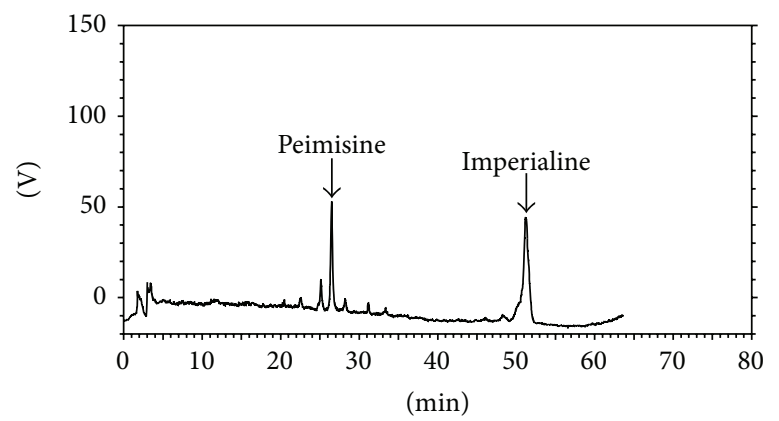

(d)

FiguRE 7: HPLC chromatograms of imperialine (a), peimisine (b), sample before treatment (c), and fraction eluted by $90 \%$ ethanol (d).

alkaloids, imperialine, and peimisine on $\mathrm{H}-103$ resin at different temperatures were well-fitted to the pseudo-first-order kinetics model, Langmuir and Freundlich isotherms models. The processes of dynamic adsorption and desorption were conducted to ensure the optimal purification parameters of the H-103 resin. To the best of our knowledge, this is the first systematic publication focusing on optimization of the extraction and enrichment for large-scale production of total alkaloids from BCFC. In conclusion, the developed methodology can also be referenced for the extraction and purification of other active compounds from herbal materials and large-scale manufacture of alkaloids of BCFC in food and pharmaceutical industry.

\section{Conflict of Interests}

The authors declare that there is no conflict of interests regarding the publication of this paper.

\section{Acknowledgment}

The authors are grateful to the Science and Technology Support Program of Sichuan Province (2011SZ0274) from the Department of Science and Technology of Sichuan Province for financial support.

\section{References}

[1] X. W. Li, J. Y. Song, J. H. Wei, Z. G. Hu, C. X. Xie, and G. A. Luo, "Natural Fostering in Fritillaria cirrhosa: integrating herbal medicine production with biodiversity conservation," Acta Pharmaceutica Sinica B, vol. 2, no. 1, pp. 77-82, 2012.

[2] X. Li, Y. Dai, and S. Chen, "Growth and physiological characteristics of Fritillaria cirrhosa in response to high irradiance and shade in age-related growth phases," Environmental and Experimental Botany, vol. 67, no. 1, pp. 77-83, 2009.

[3] L. Jin, L. Ding, G. H. Luo, Z. Chen, and Y. G. Ma, "Effects of different concentration GA3 on germination rate and esterase of Fritillaria cirrhosa seeds," Seed, vol. 28, no. 8, pp. 51-52, 2009.

[4] D. C. Hao, X. J. Gu, P. G. Xiao, and Y. Peng, "Phytochemical and biological research of Fritillaria medicinal resources," Chinese Journal of Natural Medicine, vol. 11, no. 4, pp. 330-344, 2013.

[5] S. Li, J. Liu, X. Gong, X. L. Yang, Y. G. Zhu, and Z. Cheng, "Characterizing the major morphological traits and chemical compositions in the bulbs of widely cultivated Fritillaria species in China," Biochemical Systematics and Ecology, vol. 46, pp. 130136, 2013.

[6] M. H. Chen, "Studies on Fritillaria cirrhosa D. Don and its effects of antitussive and antiasthma," Qinghai Normal University, 2008.

[7] X. Y. Yan, "Effects of ethanol extract of three kinds of Bulb Fritillariae cirrhosae on guinea pigs with allergic asthma," Sichuan University, 2005.

[8] D. G. Kang, E. J. Sohn, Y. M. Lee et al., "Effects of bulbus Fritillaria water extract on blood pressure and renal functions in the L-NAME-induced hypertensive rats," Journal of Ethnopharmacology, vol. 91, no. 1, pp. 51-56, 2004.

[9] D. D. Wang, S. Wang, Y. Feng et al., "Antitumor effects of Bulbus Fritillariae cirrhosae on Lewis lung carcinoma cells in vitro and in vivo," Industrial Crops and Products, vol. 54, pp. 92-101, 2014. 
[10] D. D. Wang, Y. Feng, Z. Li et al., "In vitro and in vivo antitumor activity of Bulbus Fritillariae cirrhosae and preliminary investigation of its mechanism," Nutrition and Cancer, 2013.

[11] Y. Li, C. Xu, Q. Zhang, J. Y. Liu, and R. X. Tan, "In vitro antiHelicobacter pylori action of 30 Chinese herbal medicines used to treat ulcer diseases," Journal of Ethnopharmacology, vol. 98, no. 3, pp. 329-333, 2005.

[12] D. Wang, J. Zhu, S. Wang et al., "Antitussive, expectorant and anti-inflammatory alkaloids from Bulbus Fritillariae cirrhosae," Fitoterapia, vol. 82, no. 8, pp. 1290-1294, 2011.

[13] D. Wang, S. Wang, X. Chen et al., "Antitussive, expectorant and anti-inflammatory activities of four alkaloids isolated from Bulbus of Fritillaria wabuensis," Journal of Ethnopharmacology, vol. 139, no. 1, pp. 189-193, 2012.

[14] C. Y. Wang, L. L. Shi, L. T. Fan et al., "Optimization of extraction and enrichment of phenolics from pomegranate (Punica granatum L.) leaves," Industrial Crops and Products, vol. 42, pp. 587-594, 2013.

[15] L. Guo, S. Y. Cho, S. S. Kang, S.-H. Lee, H.-Y. Baek, and Y. S. Kim, "Orthogonal array design for optimizing extraction efficiency of active constituents from Jakyak-Gamcho Decoction, the complex formula of herbal medicines, Paeoniae Radix and Glycyrrhizae Radix," Journal of Ethnopharmacology, vol. 113, no. 2, pp. 306-311, 2007.

[16] S.-C. Wang, H.-J. Liao, W.-C. Lee, C.-M. Huang, and T.H. Tsai, "Using orthogonal array to obtain gradient liquid chromatography conditions of enhanced peak intensity to determine geniposide and genipin with electrospray tandem mass spectrometry," Journal of Chromatography A, vol. 1212, no. 1-2, pp. 68-75, 2008.

[17] C. Q. Liu, P. Zhang, L. Liu et al., "Isolation of $\alpha$-arbutin from Xanthomonas CGMCC 1243 fermentation broth by macroporous resin adsorption chromatography," Journal of Chromatography B, vol. 925, pp. 104-109, 2013.

[18] C.-L. Lu, Y.-M. Li, G.-Q. Fu et al., "Extraction optimisation of daphnoretin from root bark of Wikstroemia indica (L.) C.A. and its anti-tumour activity tests," Food Chemistry, vol.124, no. 4, pp. 1500-1506, 2011.

[19] National Commission of Chinese Pharmacopoeia, "Pharmacopoeia of Peoples Republic of China," China Medical Science Press, vol. 1, pp. 33-35, 2008.

[20] C. Wang, S. Wang, and J. Ma, "HPLC fingerprint of Bullbus Fritillariae cirrhosae," West China Journal of Pharmaceutics, vol. 25, no. 1, pp. 61-63, 2010.

[21] Z. Wei, Y. Zu, Y. Fu et al., "Resin adsorption as a means to enrich rare stilbenes and coumarin from pigeon pea leaves extracts," Chemical Engineering Journal, vol. 172, no. 2-3, pp. 864-871, 2011.

[22] Z.-F. Zhang, Y. Liu, P. Luo, and H. Zhang, "Separation and purification of two flavone glucuronides from Erigeron multiradiatus (Lindl.) Benth with macroporous resins," Journal of Biomedicine and Biotechnology, vol. 2009, Article ID 875629, 8 pages, 2009.

[23] Z. Zhao, L. Dong, Y. Wu, and F. Lin, "Preliminary separation and purification of rutin and quercetin from Euonymus alatus (Thunb.) Siebold extracts by macroporous resins," Food and Bioproducts Processing, vol. 89, no. 4, pp. 266-272, 2011.

[24] J. N. Mi, M. Zhang, G. X. Ren, H. Y. Zhang, Y. R. Wang, and $\mathrm{P}$. Hu, "Enriched separation of protopanaxatriol ginsenosides, malonyl ginsenosides and protopanaxadiol ginsenosides from Panax ginseng using macroporous resins," Journal of Food Engineering, vol. 113, no. 4, pp. 577-588, 2012.
[25] M. T. Martin, Y. Yu, Z. Q. Cui, and Y. Zhang, "Optimization and orthogonal design of an ultrasonic-assisted aqueous extraction process for extracting chlorogenic acid from dry tobacco leaves," Chinese Journal of Natural Medicine, vol. 10, no. 4, pp. 311-320, 2012.

[26] S. K. Zhou, T. N. Bi, Y. F. Xu, R. L. Zhang, and M. J. Yang, "Extraction optimization of carbohydrate compound from Huangqi using orthogonal design," International Journal of Biological Macromolecules, vol. 58, pp. 13-17, 2013.

[27] Y. Fu, Y. Zu, W. Liu et al., "Preparative separation of vitexin and isovitexin from pigeonpea extracts with macroporous resins," Journal of Chromatography A, vol. 1139, no. 2, pp. 206-213, 2007.

[28] J. Wang, F. A. Wu, H. Zhao, L. Liu, and Q. S. Wu, "Isolation of flavonoids from mulberry (Morus alba L.) leaves with macroporous resins," African Journal of Biotechnology, vol. 7, no. 13, pp. 2147-2155, 2008.

[29] J. P. Bell and M. Tsezos, "Removal of hazardous organic pollutants by biomass adsorption," Journal of the Water Pollution Control Federation, vol. 59, no. 4, pp. 191-198, 1987.

[30] Y. Fu, Y. Zu, S. Li et al., "Separation of 7-xylosyl-10-deacetyl paclitaxel and 10-deacetylbaccatin III from the remainder extracts free of paclitaxel using macroporous resins," Journal of Chromatography A, vol. 1177, no. 1, pp. 77-86, 2008.

[31] W. Liu, S. Zhang, Y.-G. Zu et al., "Preliminary enrichment and separation of genistein and apigenin from extracts of pigeon pea roots by macroporous resins," Bioresource Technology, vol. 101, no. 12, pp. 4667-4675, 2010. 

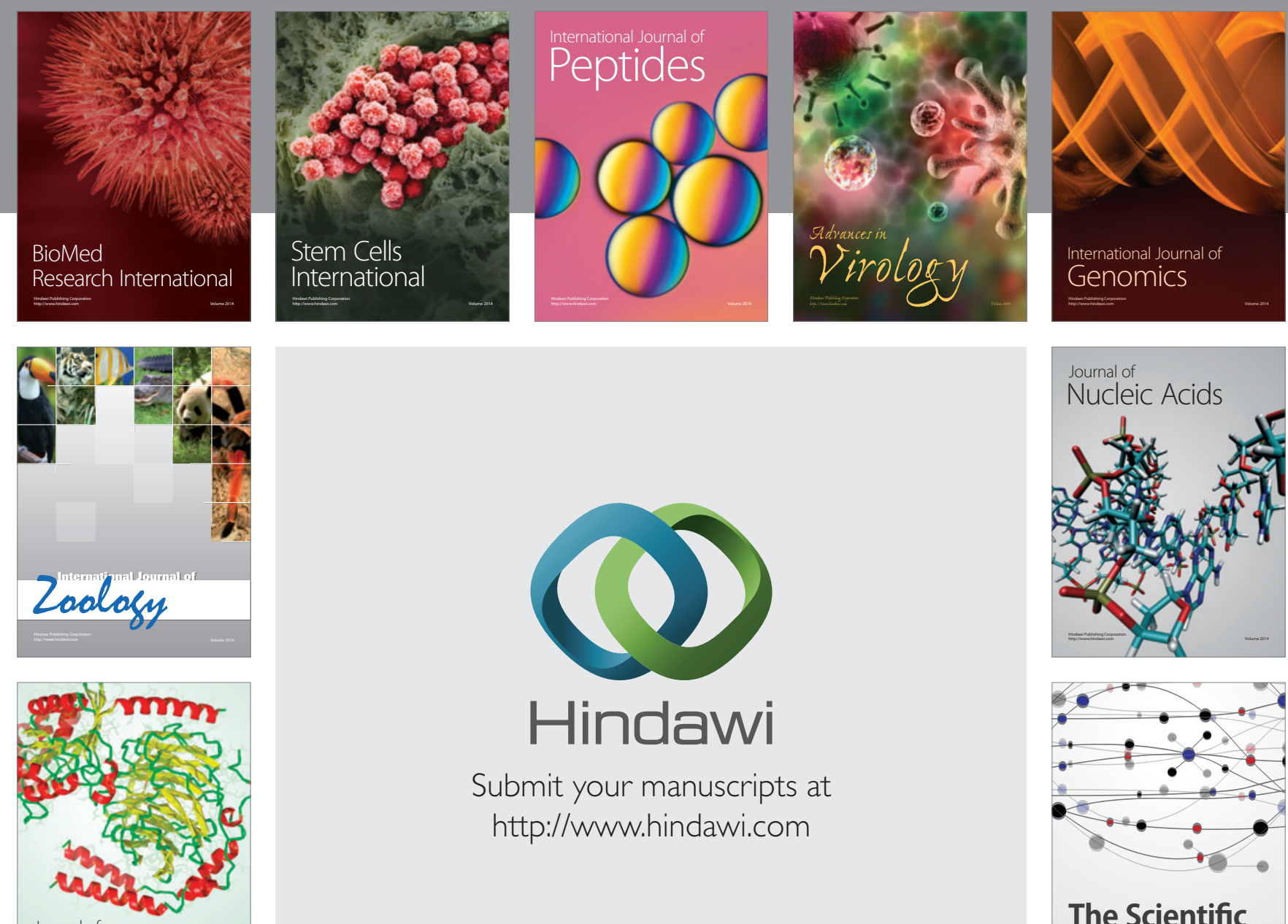

Submit your manuscripts at

http://www.hindawi.com

Journal of
Signal Transduction
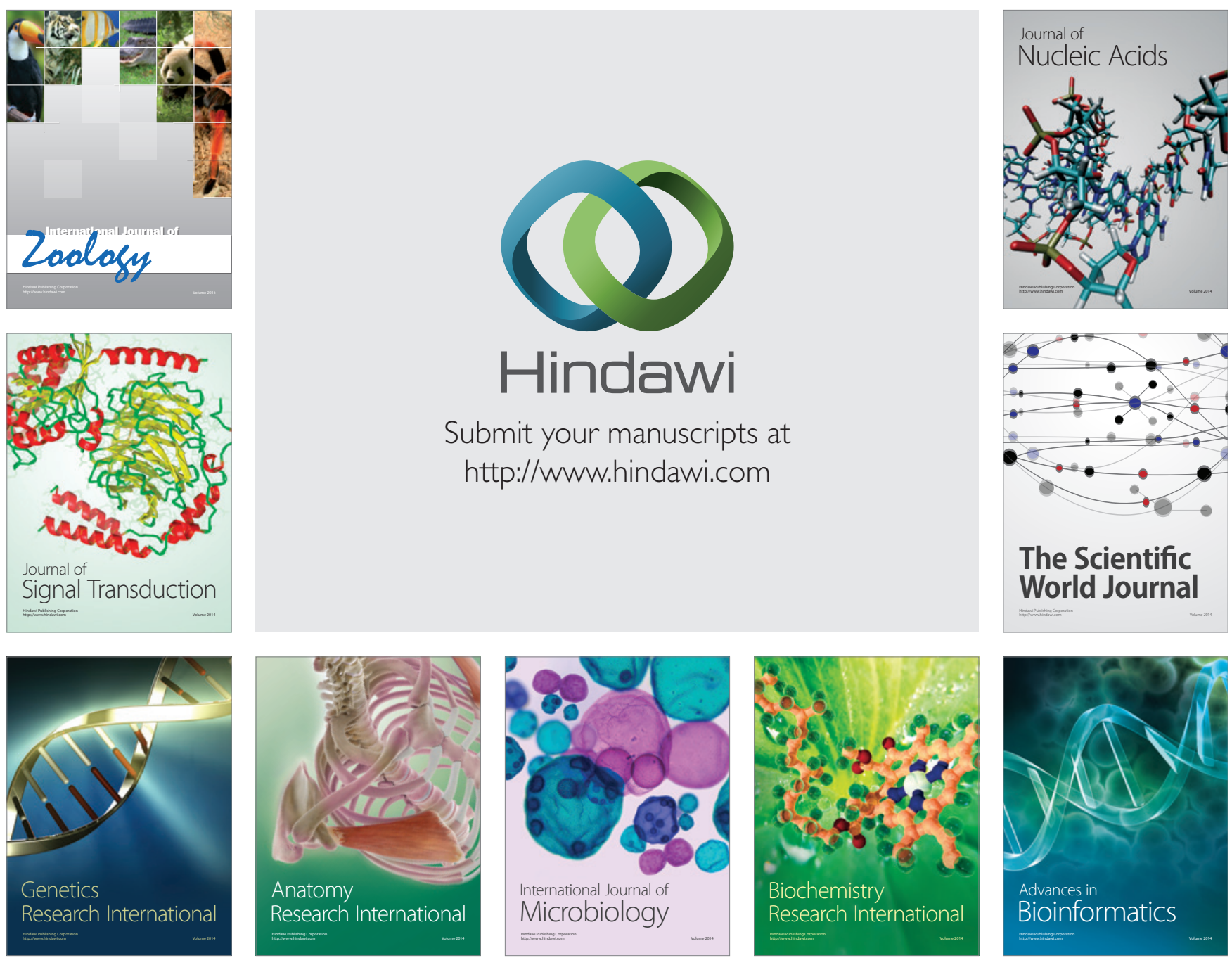

The Scientific World Journal
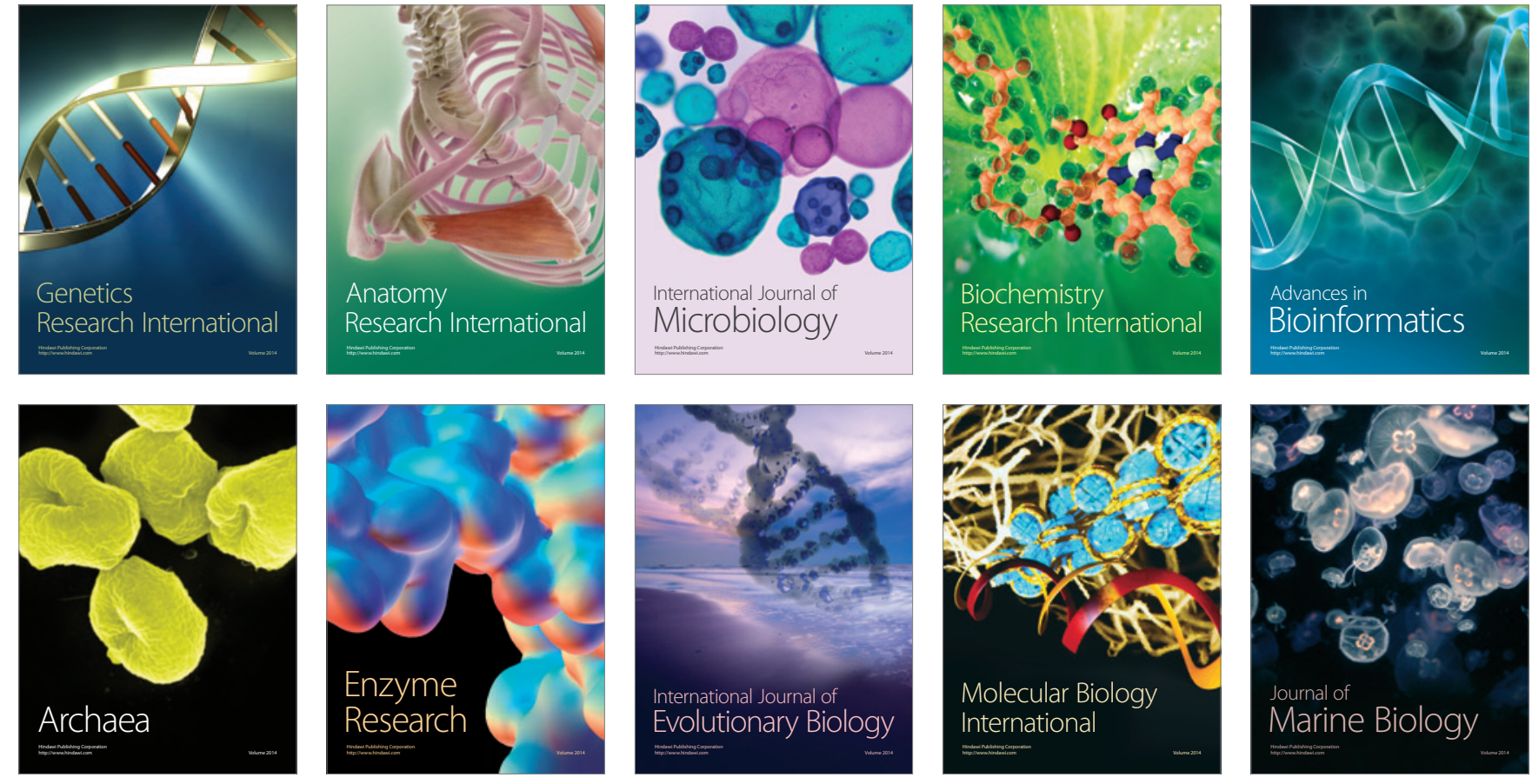